\title{
Facial Nerve Paralysis: Rare Neurological Complication of Kawasaki Disease
}

\author{
Min Sun, Xiaoyan Xu, Huan Li, Juanli Wang, Tao Wang* \\ Department of Pediatric Cardiovascular Medicine, Xi'an Children's Hospital, Xi'an, China
}

*Corresponding author: Tao Wang, Department of Pediatric Cardiovascular Medicine, Xi'an Children's Hospital, Xi'an, China

\begin{tabular}{|c|c|}
\hline ARTICLE INFO & ABSTRACT \\
\hline Received: October 21, 2020 & Citation: Min Sun, Xiaoyan Xu, Huan Li, Juanli Wang, Tao Wang. Facial Nerve Paralysis: \\
\hline Published: 㓞 October 27, 2020 & $\begin{array}{l}\text { Rare Neurological Complication of Kawasaki Disease. Biomed J Sci \& Tech Res 31(3)- } \\
\text { 2020. BJSTR. MS.ID.005100. }\end{array}$ \\
\hline
\end{tabular}

\section{Introduction}

KD Kawasaki disease (KD) or mucocutaneous lymph node syndrome was originally described by Kawasaki in 1967.It is a multisystem vasculitic disease. The diagnosis of KD is made on basis of fever at least 5 days duration with at least 4 of 5 principal criteria: changes in extremities, polymorphous erythema, bilateral conjunctival congestion, changes in lips and oral cavity, and cervical lymphadenopathy. Incomplete KD refers to patients who do not fulfill all the clinical criteria. KD is an acute panvasculitis with a striking affinity for the coronary arteries (CA) and it can affect any organ system in the body. Apart from irritability, lethargy, and aseptic meningitis, neurological complications of KD are uncommon [1]. Facial nerve paralysis (FNP) associated with KD was first reported by Murayama in 1974 [2]. FNP is a very rare manifestation of KD and was first added as one of the neurological symptoms and signs of KD in the Japanese diagnostic guidelines in 1984 [3]. FNP has been associated with a higher incidence of CA lesions and may be an indicator of more severe disease. Clinically, FNP usually presents during the convalescent phase of KD, but may develop any time from the second day of fever until>1 month after the initial KD presentation. FNP is usually transient, lasting from 2 days to 3 months, with spontaneous and complete recovery, except in 2 patients who died of cardiac complication. Treatment with intravenous immunoglobulin (IVIG) seems to shorten the time to full recovery.

\section{Case}

A 4-month-old girl was admitted to our hospital on the $20^{\text {th }}$ day of the fever with right side facial asymmetry and cough for 2 days. She had fever 20 days ago without obvious cause, and the highest temperature was up to $39.5^{\circ} \mathrm{C}$, without chill, shiver and convulsion. On day three of fever, pinhead-sized erythema rash was observed on her abdomen. A day later Then she developed left-sided deviation of the labial commissure, imcomplete closure of the right eye and obliteration of the right nasolabial fold. And she was also noticed with poor spirit and extremely irritability. She was diagnosed as bronchopneumonia and facial neuritis at local hospital and received intravenous ceftazidime for 6 days from the fever of the second day. The rash subsided day 5 from the appearance of the rash, but the fever was persistent. Two days ago, she developed cough with phlegm in throat, without wheezing. She was admitted to department of neurology in Xi'an Children's hospital with suspicious of purulent meningitis. On physical examination, the body temperature was $38.2^{\circ} \mathrm{C}$, respiratory rate was 32 breaths per minute, the pulse was regular at 145 beats per minute, blood pressure was 75/50 $\mathrm{mmHg}$, and body weight was 6.5 kilogram. General physical examination revealed presence of lung rales and hepatomegaly $(3.0 \mathrm{~cm}$ below the right coastal edge). But there was neither oral mucosa or eye changes nor enlargement of superficial lymph nodes. Edema of hands and feet, perianal desquamation was also not observed. The nervous examination revealed incomplete closure of the right eye, obliteration of the right nasolabial fold, and left-sided deviation of the labial commissure (Figure 1), other neurological examination showed no abnormality. The patient's peripheral white blood cell count was 18.1 x 109/L, with $34.6 \%$ neutrophils and $41.5 \%$ lymphocytes. The haemoglobin was 99 $\mathrm{g} / \mathrm{L}$ and the platelet count was $802.4 \mathrm{x}$ 109/L; the erythrocyte sedimentation rate(ESR) was $40 \mathrm{~mm} / \mathrm{h}$. liver and kidney function tests were normal. Serologic testing was negative for Mycoplasma 
pneumoniae, Chlamydia and Epstein-Barr virus. Chest radiograph revealed bronchitis and electrocardiogram was normal.

The initial diagnosis was suppurative meningitis. The patient was started on intravenous antibiotics, including latamoxef sodium and penicillin, while fructose sodium diphosphate, vitamin B and gangliosides was used for neurotrophic therapy. The presentation of fever, irritability and facial nerve paralysis persisted. The spiral computed tomography of brain were normal. An echocardiogram was performed showing aneurysms of the right main coronary artery(4.6mm in diameter), with diffuse dilation of left main coronary artery(2.8mm in diameter), left anterior descending coronary artery $(2.4 \mathrm{~mm}$ in diameter $)$ and left circumflex coronary artery( $2.2 \mathrm{~mm}$ in diameter) on day 2 of admission. Treatment with intravenous immunoglobulin 13 gram $(2 \mathrm{~g} / \mathrm{kg})$, aspirin $(225 \mathrm{mg} / \mathrm{d})$ was initiated immediately after incomplete Kawasaki disease (KD) was confirmed on day 3 of admission [4]. Fever and irritability resolved, the body temperature returned to normal on day 5 of admission until discharge. A total of 14 days in hospital. An echocardiogram was performed showing the right main coronary artery $(3.0 \mathrm{~mm}$ in diameter), left main coronary artery $(2.5 \mathrm{~mm}$ in diameter), left anterior descending coronary artery $(1.8 \mathrm{~mm}$ in diameter) and left circumflex coronary artery $(1.7 \mathrm{~mm}$ in diameter) on discharge. Treatment with aspirin(25mg/d) after discharge. An echocardiogram was performed showing coronary artery returned to normal 2 weeks after discharge. FNP resolved completely 1 month after diagnosis of incomplete KD (Figure 2).

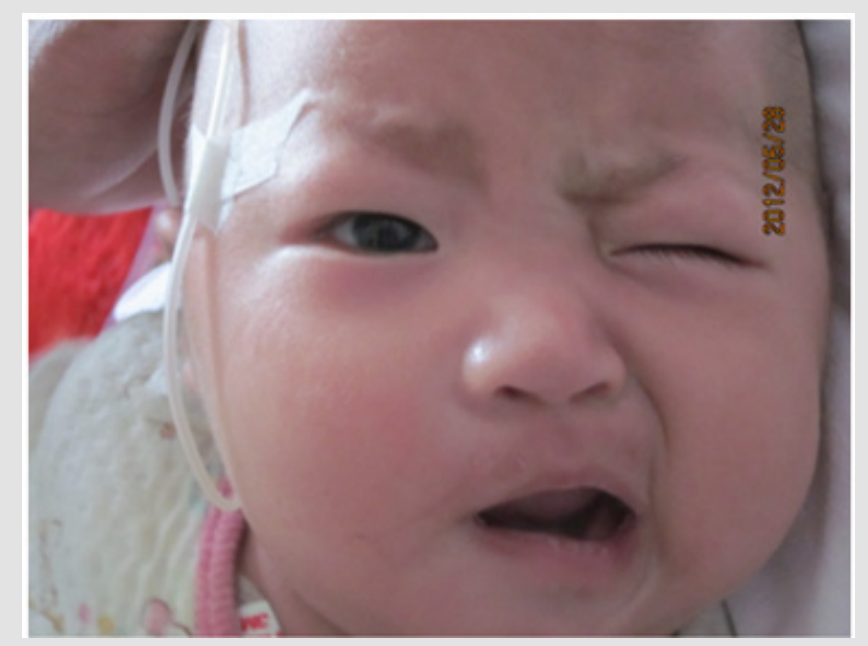

Figure 1: Four months KD infant with FNP. The infant with incomplete closure of the right eye, obliteration of the right nasolabial fold, and left-sided deviation of the labial commissure.

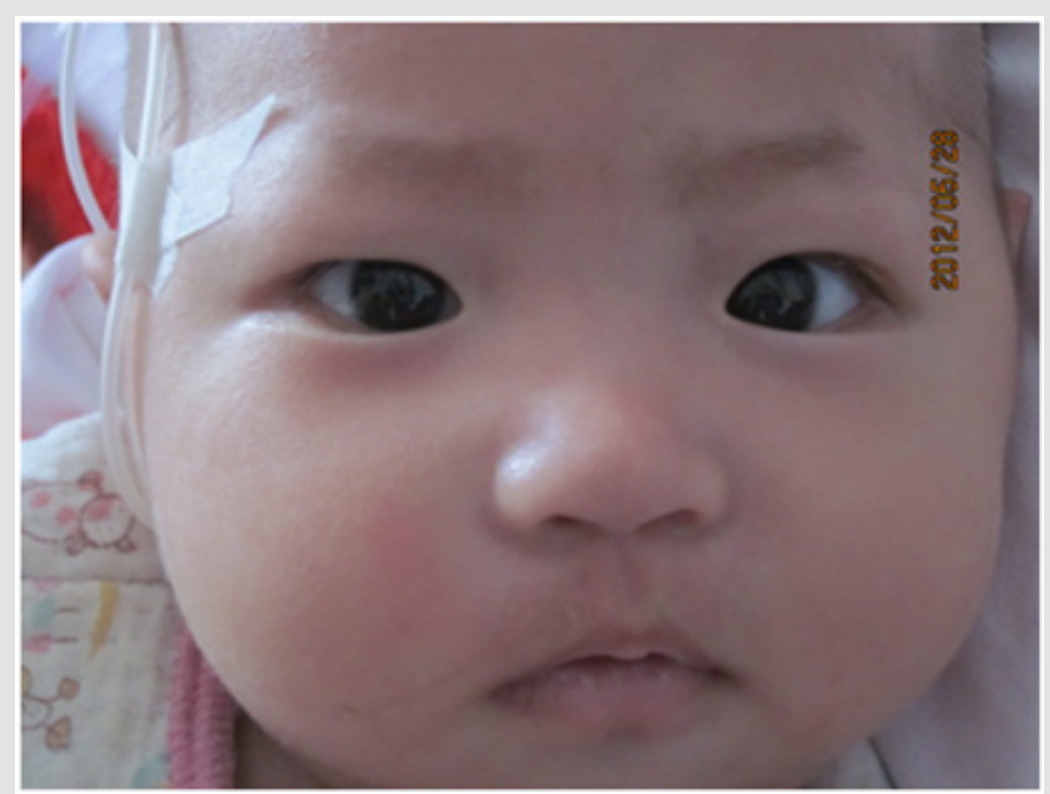

Figure 2: The same patient demonstrating complete resolution of FNP after 1 months. 


\section{Discussion}

Our patient is special in three aspects: first, her KD was incomplete(It showed repeated fever for more than 7 days, unexplained etiology, with ESR elevated and coronary artery dilatation, The body temperature returned to normal after an intravenous immunoglobulin); Secondly, the right side of FNP was affected, while previous literature reports were mostly on the left side. Thirdly, she is only 4 months old, and most reported cases were younger than 2 years old infant, the youngest one is 2 months. While $\mathrm{KD}$ is considered to be occurred mostly among 6 months to 5 years old children. Deficiencies is that the children have been fever for 20 days at the time of admission, which is more than the best time for diagnosis and treatment of KD. When the patients are suspected of KD, they should improve the examination of echocardiogram earlier, make a clear diagnosis earlier, and give treatment. Kawasaki disease with FNP mainly occurred among 2 months to 2 years old children [5]. Facial nerve paralysis is predominant in females (female: male ratio 1.4:1) and most facial nerve paralysis are unilateral, and the left side are more likely to be affected than the right [6]. To the best of our knowledge, Terence et al. first reported bilateral facial nerve paralysis in a boy with incomplete KD in 2009 [7]. And recently Xia et al. also reported bilateral facial nerve paralysis in a girl with complete KD [8]. Previously reports suggest that FNP associated with KD may indicate a higher incidence of coronary artery damage compared to KD patient without FNP [9]. LKH Poon et al reported that $54 \%$ of all cases of FNP patient without intravenous immunoglobulin developed coronary artery damage, compared with $25 \%$ of all cases of KD without intravenous immunoglobulin infusion [10]. The facial paralysis is usually transient, and mostly occurs during the convalescent phase. The palsy normally resolves spontaneously and completely, with the duration ranging from 2 days to 3 months $[6,10]$. However We think that FNP itself is probably not a risk factor for coronary artery complications, but rather FNP is simply the evidence of severe vascular inflammation in body. Thus, KD is a vasculitic disease and it can affect any organ system in the body. If KD was severe enough to cause ischemic vasculitis of the arteries supplying the facial nerve, which are thought to be the main pathophysiologic mechanisms contributing to the facial nerve palsies in KD, it may also be severe enough to lead to coronary artery damage.

In clinical practice, it is important to bear in mind the differential diagnosis of KD in any child with prolonged high fever and unexplained facial nerve palsy. Echocardiogram should be performed instantly when children are suspected of KD, which may be helpful for physician to diagnosis of $\mathrm{KD}$ at early stage and give the proper treatment. Furth more, incomplete KD is more likely to indicate severe pathological changes, and KD patients with FNP is more likely to develop into coronary artery damage. Finally, although in most cases FNP resolved spontaneously. IVIG therapy seems to improve recovery [11].

\section{Conclusion}

This case introduces the diagnosis and treatment of an incomplete Kawasaki disease complicated with FNP, and reminds you that when there is no explanation for long-term fever for more than 7 days, Clinical suspicion may be incomplete KD,Improve cardiac ultrasound diagnosis as soon as possible to avoid delay.

\section{Acknowledgement}

This work was supported by the National Natural Science Foundation of China under Grants 81974014 and 81470452.

\section{References}

1. Kawasaki T, Naoe S (2014) History of Kawasaki disease. Clin Exp Nephrol 18: 301-304.

2. Murayama J (1974) An autopsy case of acute febrile mucocutaneous lymph node syndrome with coronary embolism. Acta Paediatrica Japonica 78: 115 .

3. Kawasaki T (1985) Comment on new diagnostic guideline of Kawasaki disease. Pediatr Jpn 26: 985-993.

4. McCrindle BW, Rowley AH, Newburger JW, Newburger Jane C, Burns Anne F, et al. (2017) Diagnosis, Treatment, and Long-Term Management of Kawasaki Disease: A Scientific Statement for Health Professionals From the American Heart Association. Circulation 135(17): e927-e999.

5. Khubchandani RP, Dhanrajani A (2014) Facial nerve palsy complicating a case of Kawasaki disease. Indian J Pediatr 81(4): 406-407.

6. Rodriguez-Gonzalez M, Castellano-Martinez A, Perez-Reviriego AA (2018) Atypical Presentation of Incomplete Kawasaki Disease: A Peripheral Facial Nerve Palsy. J Emerg Med 55(1): 118-120.

7. Lim TC, Yeo WS, Loke KY, Quek SC (2009) Bilateral facial nerve palsy in Kawasaki disease. Ann Acad Med Singapore 38(8): 737-738.

8. Yu X, Liu X, Wang Y, Lu N, Wang M, et al. (2019) Kawasaki disease complicating bilateral facial nerve palsy and giant coronary artery aneurysms: A case report. Medicine (Baltimore) 98(7): e14395.

9. Stowe RC (2015) Facial nerve palsy, Kawasaki disease, and coronary artery aneurysm. Eur J Paediatr Neurol 19(5): 607-609.

10. Poon LK, Lun KS, Ng YM (2000) Facial nerve palsy and Kawasaki disease. Hong Kong Med J 6: 224-226.

11. Alves NR, Magalhães CM, Almeida Rde F, et al. (2011) Prospective study of Kawasaki disease complications: review of 115 cases. Rev Assoc Med Bras (1992) 57(3): 295-300. 
ISSN: 2574-1241

DOI: 10.26717/BJSTR.2020.31.005100

Tao Wang. Biomed J Sci \& Tech Res

(C) This work is licensed under Creative

Submission Link: https://biomedres.us/submit-manuscript.php

$\begin{array}{ll}\text { BIOMEDICAL } & \text { Assets of Publishing with us } \\ \text { RESEARCHES } & \text { Global archiving of articles } \\ \text { - Immediate, unrestricted online access }\end{array}$

